\title{
Structure of the magnetic fluid film under alternating magnetic fields
}

\author{
H.C. Yang ${ }^{\mathrm{a}, *}$, B.Y. Jeang ${ }^{\mathrm{b}}$, S.Y. Yang ${ }^{\mathrm{b}, 1}$, H.E. Horng ${ }^{\mathrm{b}}$, \\ T.P. Huang ${ }^{\mathrm{c}}$, Chin-Yih Hong ${ }^{\mathrm{c}}$ \\ ${ }^{a}$ Department of Physics, National Taiwan University, 1 Section 4 Roosevelt Road, Taipei 10617, Taiwan \\ ${ }^{\mathrm{b}}$ Department of Physics, National Taiwan Normal University, Taipei 116, Taiwan \\ ${ }^{c}$ Department of Mechanical Engineering, Da-Yeh University, Chang-Hwa 515, Taiwan
}

\begin{abstract}
Structural formation in the magnetic fluid film under alternating magnetic fields, $H_{\mathrm{ac}}$, perpendicular to the plane of the film, is investigated. For a given alternating frequency of the field, the structure in the magnetic fluid film evolves from a monodispersed state to a disordered column pattern, and then to an ordered structure when the amplitude of $H_{\mathrm{ac}}$ increases from zero. Under the application of $H_{\mathrm{ac}}$, the diameter of a magnetic column varies periodically with the same period as that of the $H_{\text {ac }}$, whereas the distance d between two neighboring columns remains fixed. This implies that the magnetic particles of columns disperse into the liquid and then agglomerate to form the original column at the same location. This process repeatedly proceeds under the application of $H_{\mathrm{ac}}$. The experimental results also show that the oscillating range of the column diameter reduces when the alternating frequency of $H_{\text {ac }}$ rises, and the averaged diameter of each column becomes larger.
\end{abstract}

(C) 2002 Elsevier Science B.V. All rights reserved.

Keywords: Magnetic fluid; Alternated field; Structure; Column size

\section{Introduction}

Pattern forming systems of magnetic fluid films under perpendicular magnetic fields have attracted scientific interest. For this area of study, a variety of pattern forming systems of magnetic fluid films have been studied, such as the geometry of agglomeration of magnetic fluids assuming a pattern of uniform cylinders of equal height and diameter [1], and quasi-twodimensional periodic lattices formed under a perpendicular magnetic field [2]. Also, the formative process of an ordered structure in magnetic fluid films has been reported $[3,4]$, in which the structural pattern evolves

\footnotetext{
*Corresponding author. Tel.: +886-2-33-66-51-77; fax: + 886-2-23-63-99-84.

E-mail address: hcyang@phys.ntu.edu.tw (H.C. Yang).

${ }^{1}$ Present address: Institute of physics, Academia Sinica, Taipei 115, Taiwan.
}

from a disordered column phase to the first-level hexagonal structure, and then to the second-level hexagonal structural pattern through a phase transition. In the above-mentioned works, the externally applied magnetic fields were driven by a DC current source. On the other hand, the work on magnetic fluids driven by an $\mathrm{AC}$ current has also been attracting the attentions of researchers, however, their works have mainly concentrated on the investigation of the rheological properties of magnetic fluid under alternating fields [5-14]. The structural forming system of a magnetic fluid film under an alternating magnetic field has remained unexplored. In this area, the structural dynamics of one-dimensional structural pattern in a magnetic fluid film has been studied [15]. However, due to the difficulty of forming a two-dimensional ordered structure in magnetic fluid films, there is no work reported on the area of a twodimensional structural forming system of magnetic fluid films. 


\section{Experimental details}

The homogeneous kerosene-based magnetic fluid $\left(\mathrm{MnFe}_{2} \mathrm{O}_{4}\right)$ with saturated magnetization of $8.7 \mathrm{emu} / \mathrm{g}$ used in this study was prepared with the co-precipitation technique. The diameter of the magnetic particle is around $120 \AA$, and the viscosity of the magnetic fluid under zero field at $25^{\circ} \mathrm{C}$ is $2.35 \mathrm{cp}$. To form a magnetic fluid thin film, the magnetic fluid was dropped into a glass cell with a thickness of $6 \mu \mathrm{m}$ and then sealed with a glass cover plate. In this experiment, a pair of solenoids, driven by an AC current source, was used to generate alternating magnetic fields perpendicularly applied to the planes of the films. The temperature was kept constant throughout each experiment by circulating cooling water around the solenoids. The photo images of the structural pattern were taken after the structural pattern reaching a periodic steady state, generally around several cycles, using an optical microscope and recorded with a personal computer. The column sizes were measured directly from these images.

\section{Results and discussion}

When a magnetic field is applied normally to the plane of a magnetic fluid film, magnetic particles agglomerate and form particle columns. As the field strength exceeds some critical value, $H_{\mathrm{h}}$, an ordered structural pattern forms with magnetic columns occupying its vortices. If the field strength is alternated with some frequency, say an AC magnetic field $H_{\mathrm{ac}}$, the structural pattern and the column size will oscillate according to the change of the field strength. Fig. 1 gives the instant images of the structural pattern under an alternating field with an alternating frequency $f_{\mathrm{H}}=0.25 \mathrm{~Hz}$ and an amplitude $H_{\max }=8.27 \mathrm{kA} / \mathrm{m}$. It clearly shows that the columns occupying the vertices of the ordered structure do not change their location in the image. However, the size of columns oscillates with a breathing frequency, $f_{\text {col }}$. The mechanism for the breathing mode of the column size lies on the fact that the magnetic field has a negative temperature effect. Thus, the increase of field strength tends to condense more particles out of the monodispersed phase. Moreover, since the structural pattern is in its ordered state, new columns cannot be formed. Therefore, the original columns will absorb more particles and grow in size. Contrarily, the decrease of the field strength causes the particles of the columns to dissolve back into the monodispersed phase and the

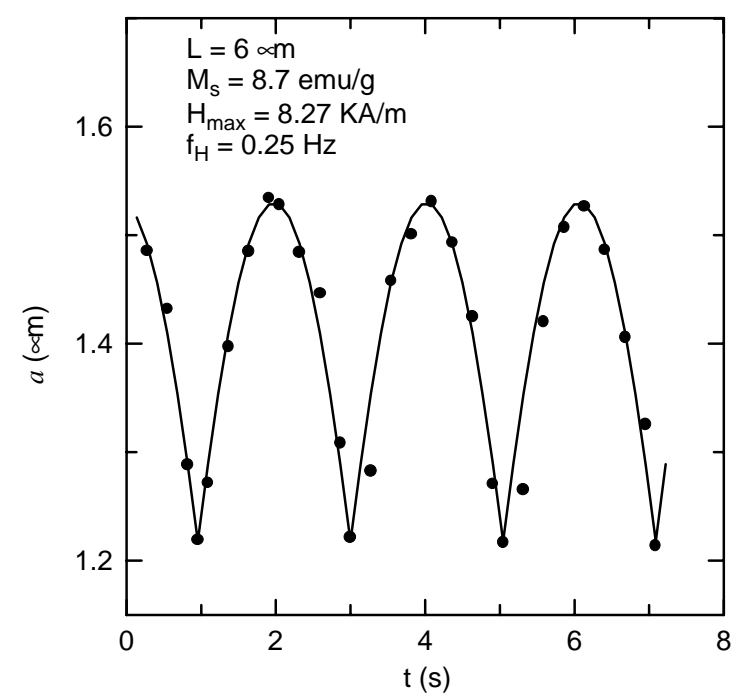

Fig. 2. Temporal evolution of the diameter of the columns in Fig. 1. It shows that the behavior of the column diameter is $a(t)=a_{\min }+\left(a_{\max }-a_{\min }\right)\left|\sin \left(2 \pi f_{\mathrm{col}} t+\phi\right)\right|$ (denoted by the solid line) with the frequency $f_{\text {col }}$ coincided with that of the driving frequency $f_{\mathrm{H}}$. (a) $\mathrm{t}=0 \mathrm{~s}$

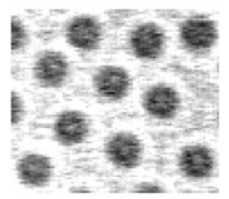

(e) $\mathrm{t}=1.90 \mathrm{~s}$

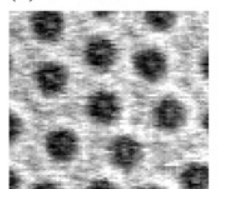

(b) $\mathrm{t}=0.68 \mathrm{~s}$

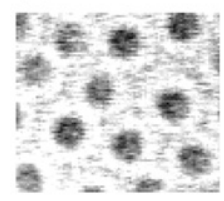

(f) $\mathrm{t}=2.45 \mathrm{~s}$

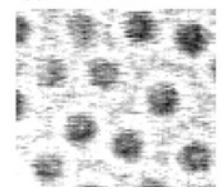

(c) $\mathrm{t}=0.95 \mathrm{~s}$

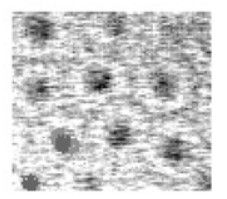

(g) $\mathrm{t}=2.99 \mathrm{~s}$

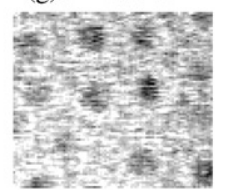

(d) $\mathrm{t}=1.49 \mathrm{~s}$

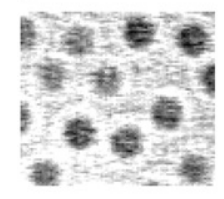

(h) $\mathrm{t}=3.27 \mathrm{~s}$

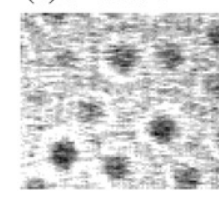

Fig. 1. Typical instant images of the magnetic columns of ordered structure in the magnetic fluid film subjected to an alternating magnetic field perpendicular to the plane of film. The alternating frequency of the magnetic field is $f_{\mathrm{H}}=0.25 \mathrm{~Hz}$ and the amplitude of the field strength is $8.27 \mathrm{kA} / \mathrm{m}$. 
Table 1

Relationship between the driving frequency of the alternating magnetic field and the breathing frequency of the magnetic column of ordered structure formed inside magnetic fluid film under perpendicular field

\begin{tabular}{lllllllll}
\hline$f_{\mathrm{H}}(\mathrm{Hz})$ & 0.200 & 0.250 & 0.500 & 1.000 & 1.500 & 2.000 & 2.500 \\
$f_{\text {col }}(\mathrm{Hz})$ & 0.198 & 0.245 & 0.489 & 0.930 & 1.544 & 1.931 & - & - \\
\hline
\end{tabular}

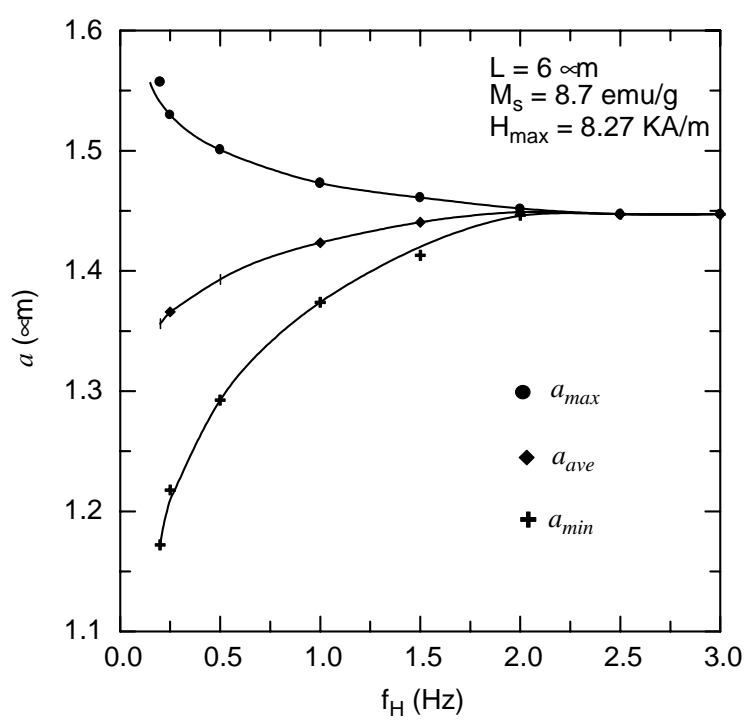

Fig. 3. Plot of the column diameter as a function of the driving frequency of the magnetic field.

column size reduces. Fig. 2 plots the time history of the column size of Fig. 1, which shows that the behavior of column size is $a(t)=a_{\min }+\left(a_{\max }-a_{\min }\right)\left|\sin \left(2 \pi f_{\text {col }} t+\phi\right)\right|$ with $f_{\text {col }}=f_{\mathrm{H}}$.

Fig. 3 gives the column sizes, which are the maximum, the averaged, and the minimum sizes, as functions of $f_{\mathrm{H}}$. At the range of $f_{\mathrm{H}}>2.3 \mathrm{~Hz}$, three curves merge and the column size remains constant regardless of the change in $f_{\mathrm{H}}$. This indicates that the response (growing) and relaxation (dissolving) times of the particles of columns are much longer than the period of the alternating field. On the other hand, in the low frequency range, three curves separated and the curve of averaged column size tends to move to a lower value. It appears that the relaxation time is shorter than the response time of columns and both of them are in the same order as the period of the driving field. Table 1 gives the values of $f_{\mathrm{H}}$ and the corresponding values of $f_{\text {col }}$.

\section{Conclusion}

When an alternating magnetic field is applied to the ordered structure of magnetic fluid film, the column size will respond according to the frequency of the driving field. At a low driving frequency range, the size of the columns behaves $a(t)=a_{\min }+\left(a_{\max }-a_{\min }\right) \mid \sin \left(2 \pi f_{\text {col }} t+\right.$ $\phi) \mid$ with a breathing frequency $f_{\text {col }}$ that is the same as the driving frequency. On the other hand, at a high driving frequency range, the size of the columns remains constant regardless of the change in the frequency of the alternating field.

\section{Acknowledgements}

This work was supported by the National Science Council of ROC under grant Nos. NSC89-2212-E-212021 and NSC89-2112-M-003-040.

\section{References}

[1] G.A. Jones, H. Niedoba, J. Magn. Magn. Mater. 73 (1988) 33.

[2] H. Wang, Y. Zhu, C. Boyd, W. Luo, A. Cebers, R.E. Rosensweig, Phys. Rev. Lett. 72 (1994) 1929.

[3] Chin-Yih Hong, I.J. Jang, H. E. Horng, C.J. Hsu, Y.D. Yao, H.C. Yang, J. Appl. Phys. 81 (1997) 4275.

[4] Chin-Yih Hong, Herng-Er Horng, F.C. Kuo, S.Y. Yang, H.C. Yang, J.M. Wu, Appl. Phys. Lett. 75 (1999) 2196.

[5] R.E. Rosensweig, Ferrohydrodynamics, Cambridge University Press, London, 1985.

[6] A.P. Gast, C.F. Zukowski, Adv. Colloid Interface Sci. 30 (1989) 153.

[7] M. Fermigier, A.P. Gast, J. Colloid Interface Sci. 154 (1992) 522

[8] T.C. Halsey, W. Toor, Phys. Rev. Lett. 65 (1990) 2820.

[9] D.R. Nelson, Physica A 177 (1991) 220.

[10] M.I. Shliomis, K.I. Morozov, Phys. Fluids 6 (1994) 2855.

[11] P.C. Fannin, B.K.P. Scaife, S.W. Charles, J. Magn. Magn. Mater. 72 (1988) 95.

[12] A. Zeuner, R. Richter, I. Rehberg, Phys. Rev. E 58 (1998) 6287.

[13] A. Zeuner, R. Richter, I. Rehberg, J. Magn. Magn. Mater. 201 (1999) 191.

[14] A. Zeuner, R. Richter, I. Rehberg, J. Magn. Magn. Mater. 201 (1999) 321.

[15] D. Wirtz, M. Fermigier, Phys. Rev. Lett. 72 (1994) 2294. 